\title{
The Shape Design Research Based on the Visual Perception Dynamics Theory
}

\author{
Kang $\mathrm{Hu}$ \\ Art and Design College \\ Wuhan University of Science and Technology \\ Wuhan, China 430065
}

\author{
Zhe Yang \\ Art and Design College \\ Wuhan University of Science and Technology \\ Wuhan, China 430065
}

\begin{abstract}
It is to offer methodological guideline for designers in product form design, to better meet the demands of users. Based on the visual perception dynamics theory, DNA molecular sequencer is selected as a design example to analyze the functional mechanism of visual perception dynamics. It confirms the pattern of visual perception dynamics in product form, analyzes the product form design method of visual perception dynamics aesthetic system, and provides a set of product form construction system and evaluation method based on visual perception dynamics.
\end{abstract}

Keywords-product design; form design; visual perception dynamics theory; dynamic pattern; DNA molecular sequencer

\section{INTRODUCTION}

In the field of product design, the form is the first medium for product to communicate with consumers. Products accurately convey the quality and philosophy contained in them through the form, and can enrich people's perception of products by using operating mechanism of visual perception in product form construction, to deepen the understanding of the form from people's visual instinct, and also provide valuable reference for designers in the process of constructing product form.

Earlier research on visual perception dynamics theory mainly focused on several classic works of Arnheim. However, the exploration of visual perception dynamics theory is mainly based on the interpretation and analysis of Arnheim's theoretical results, and a systematic methodology of visual perception guiding form generation has not been formed. In the field of product form, with the development of disciplines such as design, psychology, perceptual engineering and ergonomics, the progress of research on product form has been advanced $^{[1]}$

This paper analyzes and interprets the product form design by using visual perception dynamics theory. Taking the form design of DNA molecular sequencer as an example, this paper explores the methods of designing and evaluating the product form.

\section{METHOD}

\section{A. Functional Mechanism of Visual Perception Dynamics}

Visual perception is one of the attributes of vision, which can be divided into visual perception and visual sense. Arnheim's visual perception dynamics theory is developed on the basis of the format tower, which generalizes the "gestalt" tendency of the format tower as a "dynamic" mechanism. In terms of product form, visual perception dynamics mainly studies the visual interaction between the stimulant's configuration pattern and cognitive subject, including logical analysis and emotional interaction. The product creates itself through two levels; "shape" and "state" ${ }^{2]}$. Therefore, visual perception dynamics involves two levels in the product form, namely the formal expression and image creation.

\section{B. Product form Design Method}

For thinking and interpretation of any theory and idea, it is hoped to be inspired and translated into theoretical practice. Visual imagery is the core to communicate with the object's dynamic style and subject's subjective feeling. This section attempts to explore the ways in which visual perception dynamics forms the product form from thinking to imagery, from imagery to product.

1) Visual imagery of design form: When vision is stimulated by the object's dynamics style, it transfers to visual perception imagery with a certain "thinking ability" under the processing of visual perception, which is the bridge to connect the vision and thinking. The birth of design is limited to the determination of thinking direction, and this process requires the participation of designers and users to form a common design concept in the collision of thinking. The key to this link lies in the extraction of the visual imagery of the design form, and the design imagery is divided into sentimental imagery and rational imagery.

2) From imagery to the construction o product form: The designer gives visual perception imagery to the product form, which is the configuration arrangement of the manifestation of structural form under the guidance of the thinking intention. Only when the structural pattern that makes up the product conveys "tendentious tension" will the vision appealed by the structure of the product form to cause the exploring activities 
of the vision. When using the rational imagery of visual perception to construct the product form, the mechanical properties of the object color and the dynamic characteristics of the form are combined, geometric configuration method is taken to process the various forms of the product and create a concise and attractive product form.

\section{Aesthetic Evaluation of Dynamics Style}

The visual sense caused by the visual imagery and people's thinking reaches an isomorphism and lead to a harmonious psychological reaction. Based on the research on dynamic theory of visual perception form and the morphological analysis of the design stage of DNA molecular sequencer, several dynamic patterns commonly found in industrial products are summarized, namely inclination, deformation, contour confrontation, wedge, eccentricity, similarity attraction and repulsion.

1) Inclination: The inclination of visual dynamics in the direction is the most basic and effective way to achieve directional tension ${ }^{[3]}$. Inclination is instinctively perceived by the eye as a deviation from the standard vertical or horizontal directions. Such deviation from normal position causes the eye to produce a tendentious tension to attribute it to the standard direction, and make the form obtain perception dynamics. In "Fig. 1", the shapes of (2), (3) and (5) make a slight offset based on the standard, resulting in a small force in visual perception; shapes of (1), (4) and (6) make a large offset, causing a strong dynamic tendency to visual perception. The size of the perceived force produced by the inclined pattern is proportional to the angle deviated from the normal position ${ }^{[4]}$. Use of large-area, large-angle offset in form design is suitable for the construction of highly expressive products, making the product reveal a sense of firmness and power.
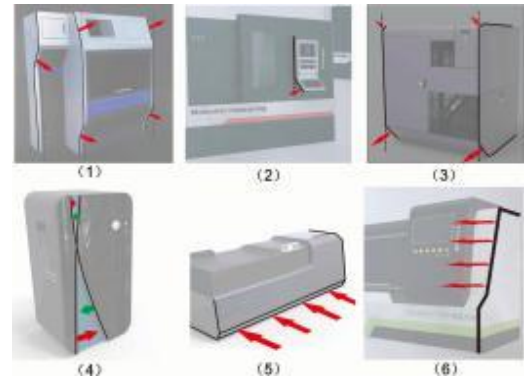

Fig. 1. Inclination

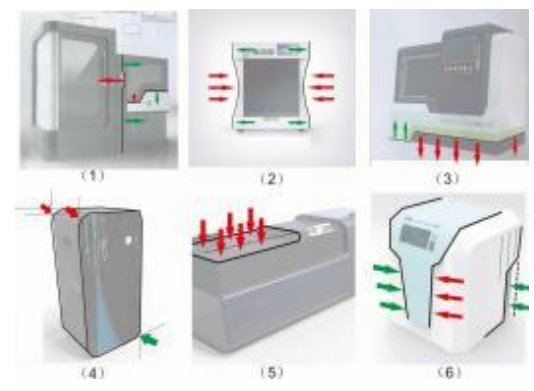

Fig. 2. Deformation
2) Deformation: Deformation is caused because the form deviates from the "exact" state that should be presented and make the visual perception produce tension. Visual instincts of human beings lean to stable shapes such as regular circles, squares or regular polygons, the force fields formed by which spread evenly around the central point. These standard shapes are the "exact" state that the visual perception thinks should be presented by the shapes. Therefore, for any variation style, the vision will generate the trend to revert it to a standard type based on standard type. In actual products, the compositional forms that make up the overall shape are seldom standard geometric shapes, with some degree of deformation based on a geometric basis more or less. In "Fig. 2", the part of overall structure of the shapes is blocked, and the visual force in (2), (4), (5) and (6) creates a reversely expanding tension for tensions balance; (1) and (3) create a complementary dynamic. Deformation gives shapes full sense of tension.

3) Contour confrontation: Vagueness and clarity caused by the visual style also lead to tendentious response of visual perception. Compared with vagueness, visual instinct leans more to clarity. Once the visual style is between clarity and vagueness, the vision produces a simplifying tendency to oppose vagueness, a common manifestation of which is contour confrontation. As shown in "Fig. 3", the overall style of the two polygons is in a steady state. However, when a partial pattern is observed, the common edge in the middle is found to be vague on all attributes. To confront vagueness, vision produces a visual force that separates the two.
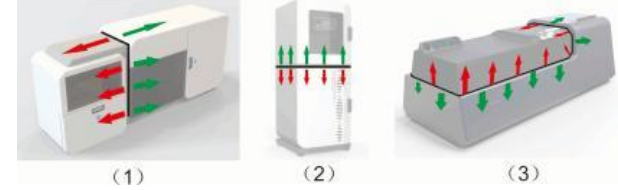

Fig. 3. Contour confrontation.

4) Wedge: Wedge shape is able to create a strong visual impact and visual tension. In this shape, the dynamic is gradually increased from the wider end to the narrower end, and decreased from the narrower end to the wider end ${ }^{[5]}$. inclination to the vision, wedge also enhances the sense of movement and activity of the form. In "Fig. 4", the wedge bodies of (1) and (3) from wide to narrow leads a bunch of downward visual force, and make the products generate a sense of stability on the ground; the structure of the bottom of (2) sets off and generates a rising force. The visual perception dynamic generated by the wedge enables the form itself to have the overall sense of conciseness and internal perception dynamics, forming a strong visual appeal in balance.
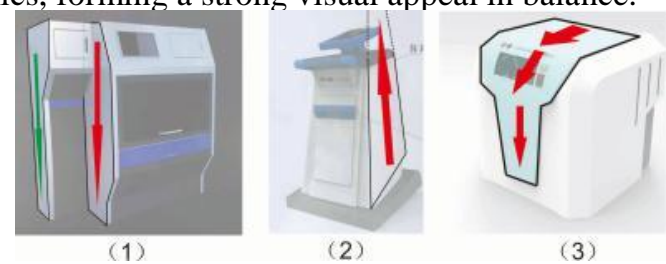

Fig. 4. Wedge. 
5) Center and eccentric: In the visual perception dynamics theory, the local form's offset relative to the overall relative position also generates perceptual dynamics, which leads to unstable state in human vision. As shown in "Fig. 5", the designer uses the eccentric force design, wherein (1) and (3) guide the user's operation in the details of the structure such as the handlebar from the perceptual level. Because it deviates from the center of the rectangular frame, there is a trend of moving to the center, and its direction coincides with the handrail's motion trail; in (2), in addition to pulling the handrail, the function keys in lower part also shows the dynamic trend of moving to the center. Using the visual instinct in product design can create more dynamic products.

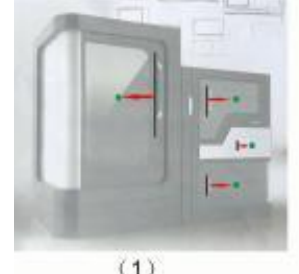

(1)

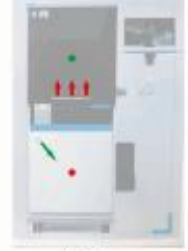

(2)

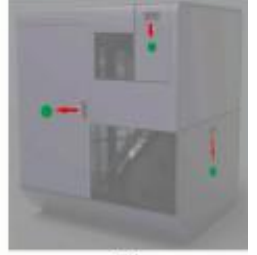

(3)
Fig. 5. Center and eccentric

6) Similarity attraction and repulsion: Similarity attraction is the dynamic to generate attraction between two identical / similar visual patterns or visual patterns perceived as a group within a certain distance, but this attraction to each other is conditional on the appropriate distance. If the two patterns are relatively too close for their own size, the two will generate dynamic for mutual repulsion in order to maintain their own independence. As shown in "Fig. 6", a trend of getting close to each other and integrating forms between the product forms shown in (1), (2), (4) and (5); however, when the two are close to a certain degree, as shown in (3) and (6), the two tend to be separated from each other because they are too close to each other. This visual dynamic of attraction and repulsion reminds designers to explore the right place for a product's form elements and create an overall visual balance.

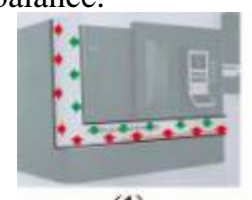

(1)

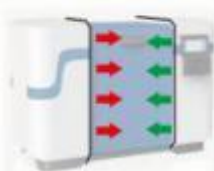

(4)

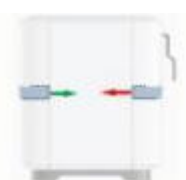

(2)

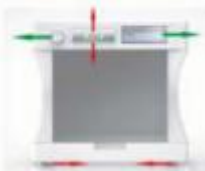

(5)

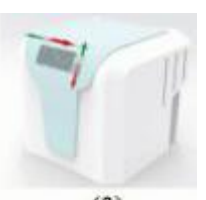

(3)

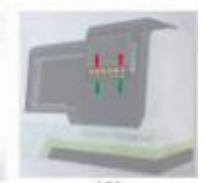

(6)
Fig. 6. Similarity attraction and repulsion.

\section{RESULT}

To verify the validity of the above model, it is used in the form design of DNA molecular sequencer. The instrument is used by researchers in scientific research institutes and universities. Focus group analysis is conducted for the users to get their perceptual expectations to DNA molecular sequencer, and then conduct design and research in the field of scientific experiment equipment, analyze the visual perception feedback of current product form, conduct related concept divergence, and transform it into a core concept to guide the later design.

\section{A. Focus Group Analysis}

Focus group analysis is a very representative method of qualitative research on things. Generally speaking, 8-12 people make up a group, and conduct in-depth discussion of a topic under the guidance of the host. In this case, the topic of discussion is the emotional orientation, attitude, focus and trend of the appearance form of DNA molecular sequencer.

Through three rounds of focus group interviews, the results of the statistics are analyzed and sorted into the following emotional perception map. Through the analysis we can see that researchers lean more to concise form, which can well balance practicality and aesthetics. This trend analysis gives designers a general direction of design entry.

\section{B. Imagery Building and Dynamic Analysis of Product Characteristics in the Same Field}

Through the interpretation of product forms with different visual feelings in the same field, designers can indirectly grasp different design languages and look for the entry point of product form in the process of weighing and optimizing.

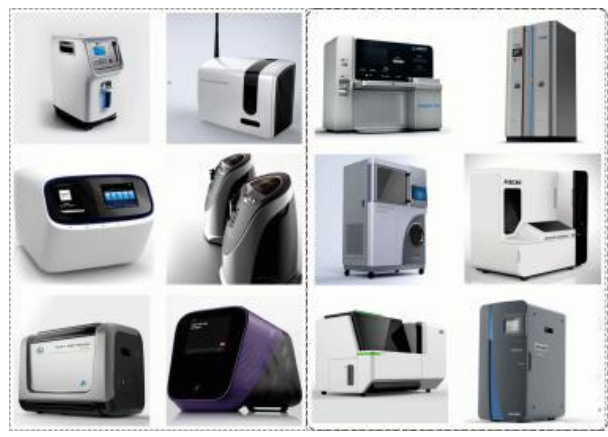

Fig. 7. Products of the same field.

"Fig. 7" shows the product form generalization and classification of current mainstream instruments in the market, by comparing the products in two styles, it can help us find some elements beneficial to later design, for example, the rounded shape on the left side is prone to intimacy, however, as an instrument, it lacks precision and cool technological sense. The instrument on the right side is in tough form through the use of a large number of linear edges, and there are rich dynamic styles between the details of the form, however, it lacks affinity and fashion sense, which fails to meet people's aesthetics, too. How to combine the advantages of both and reveal the fashion and affinity in the tough appearance has become the key to design, as well as the appeal of the above focus group research. 


\section{Product Form Building}

Analysis of DNA single molecule sequencer appearance form design based on visual perception dynamics theory shows that the appearance of the instrument needs to have an overall dynamic style and local sense of tension. Extracting the visual elements and analyzing and comparing them can make it easier to see the relationship between each solution and the design intent, and determine the final solution according to the different characteristics presented by each dynamic pattern. As shown in Fig. 8, for the analysis of the program's visual perception pattern, the consistency between the product and the user's imagery is evaluated.

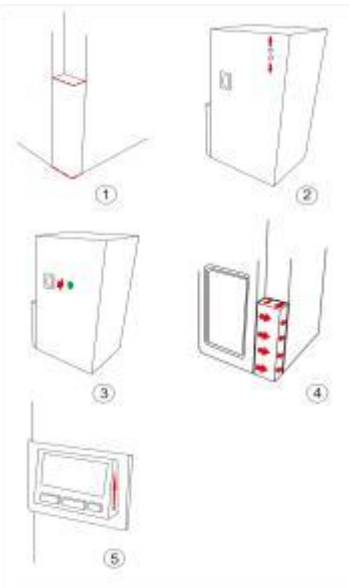

Fig. 8. Dynamic pattern.

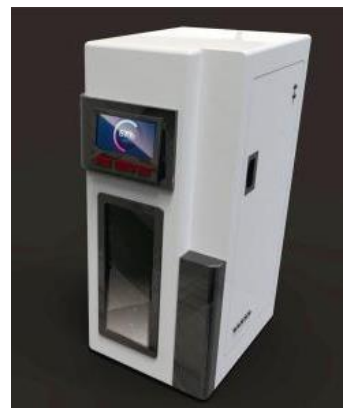

Fig. 9. Final plan rendering.

(2) (3) in "Fig. 8" well embodies the intimacy of product form. The deformation of (1) breaks the inherent stable tension balance of rectangle, creates a kind of dynamic gesture, and gives the form a full sense of tension; similarity attraction and repulsion of (2) enhances the overall sense of form, borrows simple form elements to create great visual dynamics; (3) leads to a perceptual movement that changes steadily toward balance, and breaks the presented visual harmony as the handle "intervenes" from the product side, and as the handle approaches the front, it has a tendency to move backwards and is in line with the functional track.

(4) and (5) show the technological elements inherent in instrument products. The contour confrontation of (4) generates a visual movement of separation and confrontation between the two modules, reflecting the modularized functions and form characteristics of the product; the wedge shape of (6) enhances the sense of movement and activity of the panel form of product, breaks the overall square visual balance of the product form, and forms a strong visual appeal.

As the inclining form deviates from the standard level and the plummet location, which easily leads to dynamic trends with strong visual perception, and is easy to build products with strong performance, and reflect the performance and experience of the product. This style contradicts the conclusions of the focus group analysis, so the dynamic style form design is given up.

\section{CONCLUSION}

Based on the form dynamics theory, the form dynamics is taken as the core idea and there is a new development and improvement in the combination with product form. Starting from the concept of form dynamics and product form, this paper analyzes the relationship between the two and summarizes the form performance and imagery creation of product form. On this basis, the author summarizes the six basic styles of common visual perception dynamics, and analyzes its specific application to the form of products based on examples.

Although this paper attempts to establish a set of product form generation system based on the visual perception form dynamics, it analyzes the role of visual perception dynamics theory from product form, rather than from the aspects of product color and product material. The products selected belong to scientific instrument products, with certain imitations, which needs to be further enriched.

\section{REFERENCES}

[1] Huang Kai. Product form design research based on the sentimental imagery [C]. Lanzhou University of Technology, 2012

[2] Wang Jun. Shape and state in design [J]. Art Panorama, 2014,05

[3] Zhang Wenbin. Manifestation mode of Arnheim's visual dynamics [J]. Art and Time (issue in the first third of a month), 2014,09

[4] Liu Yin, Lou Yu. Research on cause of the dynamic form of Baroque art's visual perception [J] Qilu Realm of Arts, 2014,04

[5] Ma Lianfei. Arnheim's visual form dynamic theory and dynamic comic creation [J]. Journal of Anhui Normal University (Humanities and Social Sciences Edition), 2016,05 